\title{
Publisher Correction: A systems approach identifies HIPK2 as a key regulator of kidney fibrosis
}

Yuanmeng Jin, Krishna Ratnam, Peter Y. Chuang, Ying Fan, Yifei Zhong, Yan Dai, Amin R. Mazloom, Edward Y. Chen, Vivette D'Agati, Huabao Xiong, Michael J. Ross, Nan Chen, Avi Ma'ayan and John Cijiang He

Correction to: Nature Medicine https://doi.org/10.1038/nm.2685, published online 11 March 2012.

In the version of this article initially published, the Supplementary Information file lacked the Supplementary Methods section. The correct file that includes Supplementary Methods is provided here.

\section{Additional information}

Supplementary information The online version contains supplementary material available at https://doi.org/10.1038/s41591-021-01427-1.

Published online: 20 July 2021

https://doi.org/10.1038/s41591-021-01427-1

(c) The Author(s), under exclusive licence to Springer Nature America, Inc. 2021 